\title{
Spondylodiscitis developing in a young man - diagnostic and therapeutic difficulties
}

\author{
Desislava Kalinova, Rasho Rashkov \\ Clinic of Rheumatology, UMHAT St. Ivan Rilski University Hospital, Sofia, Bulgaria
}

\begin{abstract}
Infectious spondylodiscitis is characterized by vertebral osteomyelitis, spondylitis, and discitis. $\mathrm{Pa}$ tients present with persistent low back pain, fever, or neurological findings. Diagnosis is made with a combination of clinical, radiological, and laboratory findings. Magnetic resonance tomography (MRI) has high sensitivity and specificity in diagnosis and differentiation of the type of spondylodiscitis and may reveal signs of spondylodiscitis even in very early stages. Infectious spondylodiscitis responds to antimicrobial therapy well if diagnosed early before development of neurological deficit and requirement of surgical intervention. We present a clinical case of spondylodiscitis developing in a young immunocompetent man without any predisposing factors.
\end{abstract}

Key words: Staphylococcus aureus, infectious spondylodiscitis, magnetic resonance tomography.

\section{Introduction}

Infectious spondylodiscitis is a rare but serious disease of the intervertebral disc, which may also involve peri-vertebral structures and may lead to extensive destruction and general symptoms and neurological deficits. Patients present with a variety of symptoms including back pain, fever, nausea, and weight loss. There is often a delay in diagnosis due to the nonspecific nature of symptoms [1, 2]. Spondylodiscitis occurs secondary to a variety of causes, most notably bloodstream infections (e.g. Staphylococcus aureus) and after surgery and endoscopic procedures [3]. Subject with immunodeficiency, those with chronic diseases and immunosuppressive therapy are at particular risk for spondylodiscitis. The most commonly found pathogen responsible for infectious spondylodiscitis is Staphylococcus aureus, but coagulase-negative staphylococci, Streptococcus species, Pseudomonas aeruginosa, Escherichia coli, and fungi such as Candida albicans are also regularly found [3].

To confirm a diagnosis of infectious spondylodiscitis the basic tests in such cases are used: blood cultures, magnetic resonance tomography (MRI) scans, and vertebral biopsies [1]. MRI scan has proven to be the modality of choice for most physicians, with high sensitivity even early in the disease course [4].

The recommended treatment is administration of intravenous antibiotics initially (2-4 weeks), then oral (6-12 weeks). The main variation seems to be in choice, route of administration, and duration of antibiotic therapy [2]. Evidence suggests that patients should be treated for at least six weeks with antibiotics and preferably 12 weeks [5]. Due to the lack of randomised controlled trials there is still no high-level evidence on which treatment regimen provides the best outcome in patients with spondylodiscitis.

\section{Case report}

The male, 35-year-old patient was admitted to hospital with a tender and swollen left ankle, pain and limited movements in the shoulders, as well as low back pain (LBP) and pain in the area around the lumbar vertebrae (L1-L4). Three intramuscular injections with betamethasone were made before admission to the hospital.

The laboratory results were as follows: haemoglobin $139 \mathrm{~g} / \mathrm{l}$ (n: 135-180); erythrocytes $4.6 \times 10^{12}$ (n: 4.4-5.9); leucocytes $11.76 \times 10^{9}(\mathrm{n}: 3.5-10.5)$; platelets $166 \times 10^{9}(\mathrm{n}: 130$ 360); C-reactive protein $102 \mathrm{mg} / \mathrm{l}(<5 \mathrm{mg} / \mathrm{l})$; erythrocyte

Address for correspondence:

Desislava Kalinova, Clinic of Rheumatology, UMHAT St. Ivan Rilski University Hospital, 13 Urvich St., 1612 Sofia, Bulgaria,

e-mail: d_kalinova666@abv.bg

Submitted: 2.07.2018; Accepted: 12.10.2018 
sedimentation rate (ESR) $78 \mathrm{~mm} / \mathrm{h}(\mathrm{n}:<15 \mathrm{~mm} / \mathrm{h})$; AspAT $81 \mathrm{U} / \mathrm{l}(\mathrm{n}:<32 \mathrm{U} / \mathrm{l})$; AlAT $49 \mathrm{U} / \mathrm{l}(\mathrm{n}$ : < $33 \mathrm{U} / \mathrm{l})$; GGTP $111 \mathrm{U} / \mathrm{l}(\mathrm{n}:<40 \mathrm{U} / \mathrm{l})$; AP $141 \mathrm{U} / \mathrm{l}(\mathrm{n}:<105 \mathrm{U} / \mathrm{l})$; total bilirubin $31.9 \mu \mathrm{mol} / \mathrm{l}(\mathrm{n}:<21 \mu \mathrm{mol} / \mathrm{l})$; direct bilirubin $12.3 \mu \mathrm{mol} / \mathrm{l}$ $(\mathrm{n}:<8.5 \mu \mathrm{mol} / \mathrm{l})$. Also laboratory tests revealed rheumatoid factor positivity for IgM, IgA and IgG classes of immunoglobulin (n: 243.8; 182.1 and $95.1 \mathrm{U} / \mathrm{ml}$ respectively), as well as anti-citrullinated peptide antibodies (ACPA) $93.6 \mathrm{U}$ (normal range < $20 \mathrm{U}$ ), and anti-mutated citrullinated peptide antibody (anti-MCV) $20.8 \mathrm{U} / \mathrm{ml}$ (normal range $<20 \mathrm{U} / \mathrm{ml}$ ). HLA-B27 was negative.

Ultrasound examination of the left ankle showed synovitis with positive power Doppler signal. Ultrasound of the small joints of the hands did not present pathological features. $X$-ray of sacroiliac joints revealed a rough right sacroiliac joint (Fig. 1). X-ray of ankles, hands and the thoracolumbar part of the spine with sacroiliac joints did not show pathological changes.

Cultures (blood culture, sterile urine) were negative for infectious organisms. Blood serological tests for Chlamydia trachomatis antibodies (IgG, IgM, IgA class) and polymerase chain reaction (PCR) testing for Chlamydia trachomatis in a urine specimen were negative. The $X$-ray of the lungs and the ultrasound examination of the abdomen also did not reveal abnormalities.

The patient had no comorbidities or family history of arthritis, but he was diagnosed with hepatitis A virus in November 2016.

Analysis of symptoms and additional tests were taken into consideration diagnosis as follows:

- seronegative spondyloarthropathy,

- rheumatoid arthritis,

- undifferentiated arthritis.

Seronegative spondyloarthropathy (SpA) was initially diagnosed - inflammatory back pain, onset before 45 years of age, in the X-ray image of the right sacroiliac joint there was a suggestion of sacroiliitis, despite the absence of the HLA-B27 antigen. MRI of the spine or sacroiliac joints had not been performed at this stage.

The patient did not fulfil the criteria for classification (ACR/EULAR classification criteria from 2010) of rheumatoid arthritis (RA); he had ACPA antibodies and rheumatoid factor (RF), but had only one joint involvement.

Undifferentiated arthritis was considered but LBP and arthritis strongly suggested an early stage of SpA, even though HLA-B27 was not present.

Corticosteroid was applied locally in the left ankle. Therapy with sulfasalazine $2 \mathrm{~g}$ /daily and a non-steroidal anti-inflammatory drug (NSAID) was initiated.

Also the patient consulted a gastroenterologist who concluded that the patient had protracted hepatitis A infection. Therapy with ademetionine $500 \mathrm{mg}$ twice daily was prescribed.
One month later the patient presented to the rheumatologist with fever, low back pain, pain in vertebrae and spinous processes located in the lumbar region of the spine. Laboratory tests showed high inflammatory serum markers: ESR $84 \mathrm{~mm} / \mathrm{h}$ and CRP $51.2 \mathrm{mg} / \mathrm{l}$. Computed tomography of the spine demonstrated destruction of the L1-L2 disk space with the adjacent $L 1$ and $L 2$ vertebral bodies (the image corresponded with spondylodiscitis), as the contiguous iliopsoas muscle was affected (Fig. 2).

Blood cultures were negative. The Mantoux test and QuantiFERON (QFT) test for tuberculosis or latent tuberculosis infection were performed and were negative. Sulfasalazine treatment was discontinued. Therapy with ceftriaxone $4 \mathrm{~g}$ /daily and lincomycin $600 \mathrm{mg}$ three times daily intravenous application was initiated. The antibiotic therapy was discontinued after 21 days after MRI examination of the lumbar spine, which showed destruction of the L1-L2 and L3-L4 disk spaces with the adjacent vertebral bodies. The T2-weighted MR image demonstrated discitis and osteomyelitis persisting as an abscess in the structure of right iliopsoas muscle (Fig. 3).

Methicillin-resistant Staphylococcus aureus (MRSA) was detected in the next three consecutive blood cultures. The patient consulted a neurosurgeon who stated that there was considered an indication for surgery if the patient did not show improvement on treatment with targeted antibiotics. Antibiotic therapy with teicoplanin $400 \mathrm{mg}$ daily and levofloxacin 500 mg daily intravenous application was started and was applied for six consecutive weeks. Rifampicin $300 \mathrm{mg}$ daily was prescribed for three months. Intravenous immunoglobulins were applied in a dose of $400 \mathrm{mg} / \mathrm{kg}$ in three contiguous months.

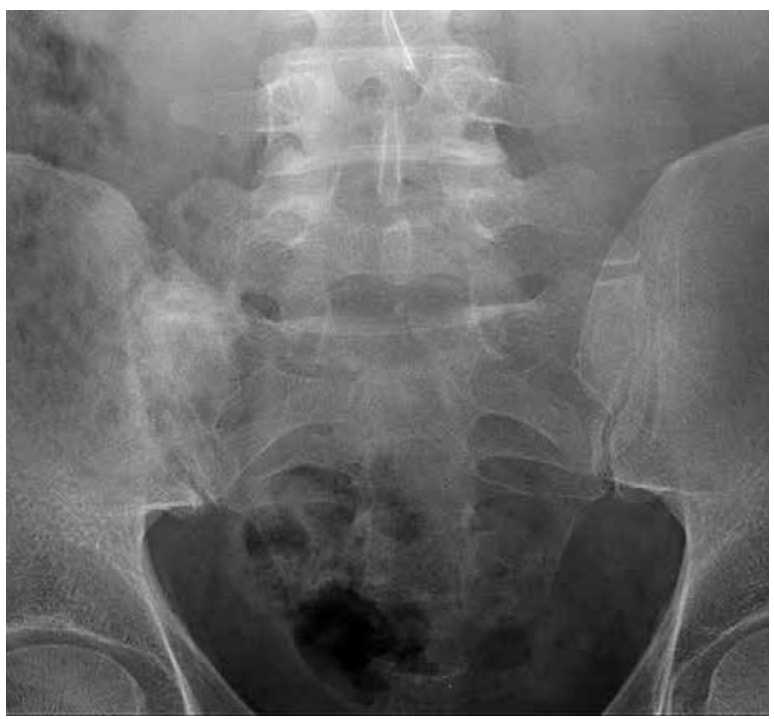

Fig. 1. X-ray of sacroiliac joints manifested rough right sacroiliac joint. 

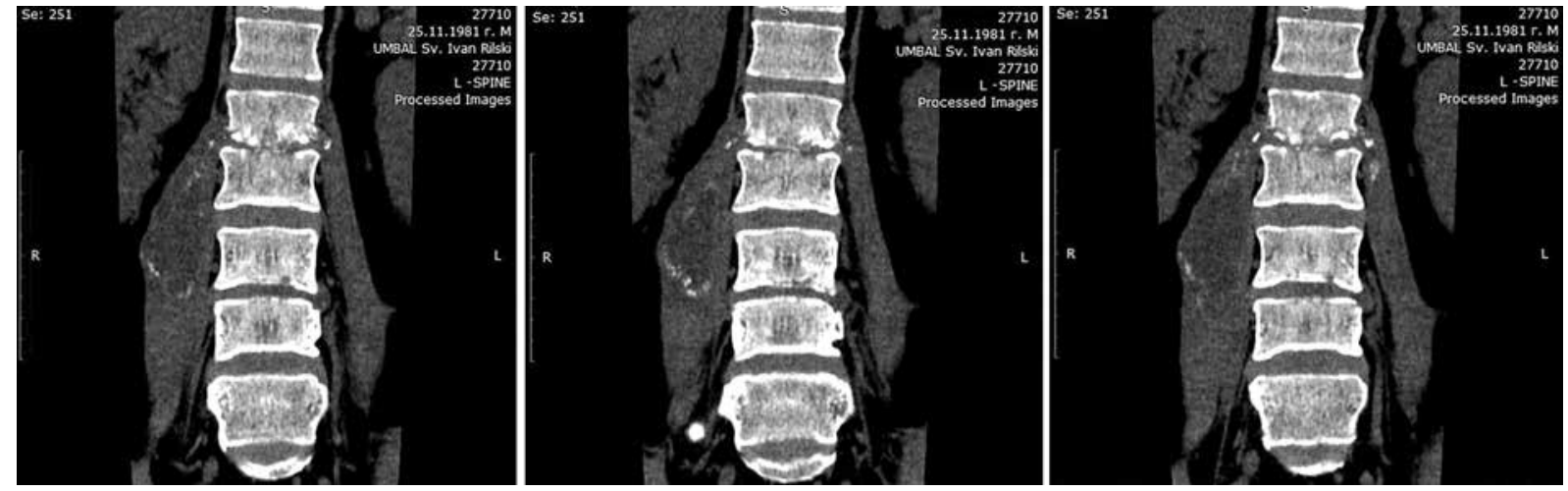

Fig. 2. Computed tomography of the spine demonstrated destruction of the L1-L2 disk space with the adjacent L1 and L2 vertebral bodies, as the contiguous $\mathrm{m}$. iliopsoas was affected.
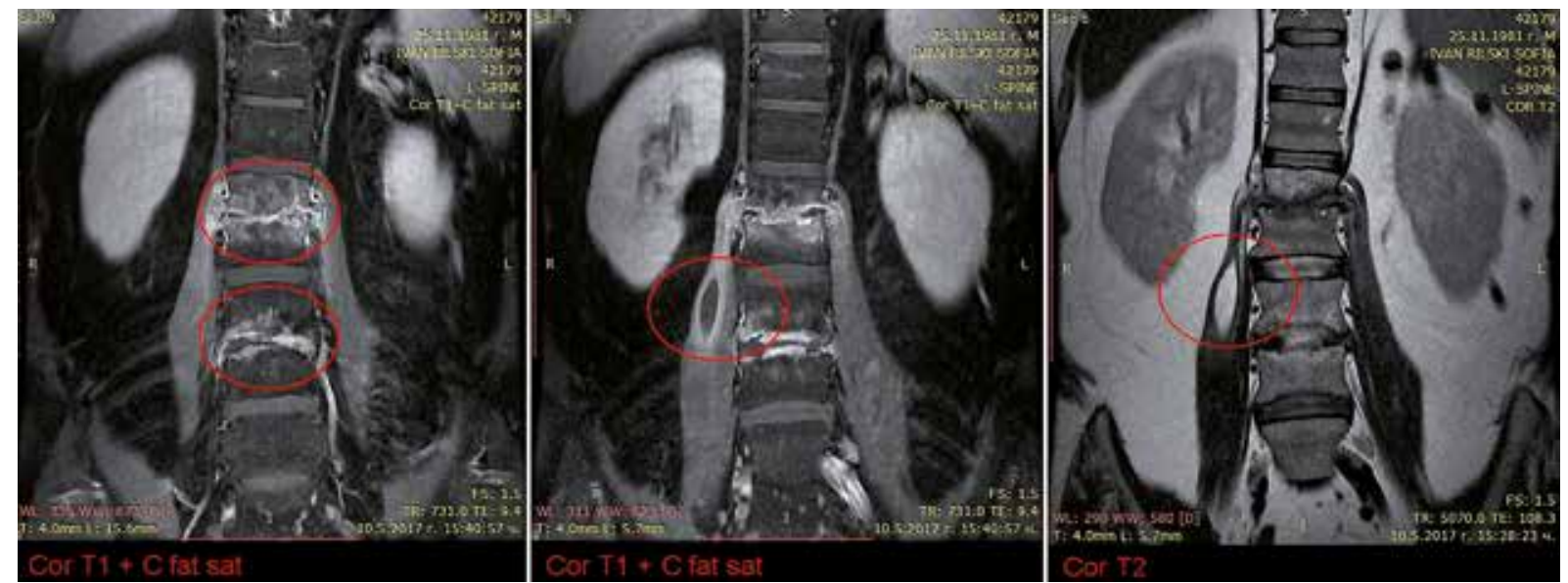

Fig. 3. MRI of the spine (May 2017) showed destruction of the L1-L2 and L3-L4 disk spaces with the adjacent vertebral bodies, as an abscess in the structure of the right $\mathrm{m}$. iliopsoas.

After the end of the antibiotic course the laboratory results were as follows: haemoglobin $161 \mathrm{~g} / \mathrm{l}$ (n: 135-180); erythrocytes $5.25 \times 10^{12}(\mathrm{n}: 4.4-5.9)$; leucocytes $10.5 \times 10^{9}$ (n: 3.5-10.5); platelets $181 \times 10^{9}$ (n: 130-360); C-reactive protein $4.6 \mathrm{mg} / \mathrm{l}(\mathrm{n}:<5 \mathrm{mg} / \mathrm{l})$; erythrocyte sedimentation rate (ESR) $28 \mathrm{~mm} / \mathrm{h}(\mathrm{n}:<15 \mathrm{~mm} / \mathrm{h})$; AspAT $39 \mathrm{U} / \mathrm{l}$ (n: < $32 \mathrm{U} / \mathrm{l})$; AlAT $22 \mathrm{U} / \mathrm{l}(\mathrm{n}:<33 \mathrm{U} / \mathrm{l})$; GGTP $69 \mathrm{U} / \mathrm{l}$ (n: < $40 \mathrm{U} / \mathrm{l})$; AP $105 \mathrm{U} / \mathrm{l}(\mathrm{n}:<105 \mathrm{U} / \mathrm{l})$; total bilirubin $20.5 \mu \mathrm{mol} / \mathrm{l}(\mathrm{n}:<21 \mu \mathrm{mol} / \mathrm{l})$; direct bilirubin $11.9 \mu \mathrm{mol} / \mathrm{l}$ $(<8.5 \mu \mathrm{mol} / \mathrm{l})$. There was no deterioration of the liver function test, but the liver enzymes and bilirubin remaining elevated.

Three months later control MRI was performed and visualized in the structure of the bodies of L1, L2, L3, L4 vertebrae and the adjacent disc surfaces irregular shaped areas with characteristic bone marrow oedema and fat bone marrow transformation. The height of the L1-L2, L3-L4 vertebrae decreased with the pathologically increased signal intensity. Zones of changed signal in- tensity were visualized in the structure of the two psoas muscles, at level L1-L2, with high signal intensity in T2 dependent sequences (post-inflammatory changes).

\section{Discussion}

Low back pain is a symptom of various diseases. The aetiology of LBP may be difficult to determine at times because of the number of diverse anatomic structures located in or near this area of the body. The etiological characterization of LBP is a process that requires a propaedeutic approach that includes the clinical history, physical and complementary examinations. The approach to low back pain of mechanical origin, and other less common reasons such as those with a neuropathic component or resulting from aseptic and septic inflammation (spondylodiscitis) or neoplasia was developed [6].

Spondylodiscitis may be bacterial, fungal, parasitic, or mycobacterial. The most frequent pathogen is Staphylococcus aureus, which is isolated in $30-50 \%$ of non-tuber- 
culosis spondylodiscitis cases [7, 8]. Predisposing factors for infectious spondylodiscitis are foci of local infections, remote infections, AIDS, alcohol use, chronic renal failure, diabetes mellitus, intravenous drug use, malignancy, and history of spinal trauma, catheter-associated infections, surgical interventions, infective endocarditis, urinary tract infections, and immunocompromised states [9]. In 20\% of cases there is no predisposing factor [10], as in the present case. We present a clinical case of spondylodiscitis, developing in a young immunocompetent man without any predisposing factors.

Infectious spondylodiscitis usually presents with fever, low back pain, local tenderness in the part of affected vertebra, neurological deficit, and high inflammatory markers (ESR, CRP). Symptoms and signs of spondylodiscitis are often non-specific, so diagnosis of infectious spondylodiscitis is difficult in many patients. Spondylodiscitis should be suspected when patients present with fever together with low back pain, and clinical, laboratory and radiological evaluations should be done $[8,10]$. In our case the patient presented with low back pain, as only $X$-ray of the sacroiliac joints and thoracolumbar area of the spine was performed in the first hospitalization. However, as is known, classic radiological imaging does not reveal infectious lesions in the early stages on the disease.

Evaluation of patients with low back pain by MRI appears to be the method of choice for detecting lesions of spondylodiscitis particularly of infectious origin and at the initial stages, showing as bone marrow oedema and increased signal of the intervertebral disc and the verte- bral body after gadolinium administration [11, 12]. Contrast-enhanced MRI is the modality of choice in clinical practice. Small studies investigating the value of magnetic resonance imaging in diagnosing spondylodiscitis showed a sensitivity of $82-96 \%$, specificity of $85-93 \%$ and accuracy of 81-94\% [13]. In the next hospitalization although there were no predisposing factors in a young immunocompetent man, because of the presence of low back pain, fever, and persistent high ESR and CRP level, CT and MRI of the lumbar spine were performed, and spondylodiscitis was diagnosed, but only the positive results of the microbiological examination of blood cultures clearly confirmed infectious inflammation. Blood cultures should be performed because in $60 \%$ of spondylodiscitis cases they are positive [14]. However, in about $40 \%$ of cases of infectious spondylodiscitis the pathogen remains undetected on the basis of blood cultures, and in some cases only the culture of the material obtained from biopsy or surgery allows its discovery.

Analysing the case, it may be assumed that ankle inflammation was aseptic and could accompany an existing infection in another area of the body. It is assumed that glucocorticoids administration having a positive effect on the treatment of arthritis could not be the causative agent of infectious disc inflammation, looking at the chronology of events in this case. Lack of efficacy of NSAID and SF treatment in the area of spinal pain may indicate its unusual cause. In the case of inflammatory back pain and involvement of the peripheral joint, more undifferentiated spondyloarthritis was suggested,

Table I. Comparison of symptoms in described case and seronegative spondyloarthropathy

\begin{tabular}{|c|c|}
\hline Non-infectious spondylodiscitis & Infectious spondylodiscitis in present case \\
\hline Onset $<40$ years of age & Onset $<40$ years of age \\
\hline LBP lasting more than 3 months & LBP lasting more than 2 weeks \\
\hline Improvement after motion & Persistent pain, enhanced during activity \\
\hline Pain of the spine & Localized pain (point/s) \\
\hline Morning stiffness & Chronic and increasing stiffness \\
\hline Involvement of sacroiliac and peripheral joints & Involvement of peripheral joint (coexisting arthritis?) \\
\hline General symptoms: & General symptoms: \\
\hline Fever & Fever \\
\hline Weakness, tiredness & Weakness, tiredness \\
\hline Weight loss & Weight loss in this particular case did not occur \\
\hline ESR and CRP normal or elevated & ESR and CRP elevated \\
\hline Blood cultures negative & Blood cultures positive \\
\hline $\begin{array}{l}\text { X-ray examination - syndesmophytes, sacroiliitis, squaring } \\
\text { of vertebral bodies }\end{array}$ & X-ray did not reveal changes at an early stage \\
\hline $\begin{array}{l}\text { MRI sacroiliac joints active inflammation, spondylitis, bone } \\
\text { morrow oedema, discitis - aseptic origin }\end{array}$ & $\begin{array}{l}\text { MRI - bone marrow oedema, narrowing spaces between vertebral } \\
\text { bodies and destruction of vertebral body, inflammatory infiltration } \\
\text { on surrounding spinal and muscle structures with abscesses }\end{array}$ \\
\hline
\end{tabular}


which narrowed down the diagnosis to unclassified arthritis. In Table I a comparison of patient symptoms and spondyloarthropathy symptoms is presented. It cannot be ruled out that previous and protracted infection with hepatitis A virus was associated with a decrease in resistance to other infections, including bacterial infections. Table I presents some similarities between SpA and symptoms in the case of the described patient.

Combined ${ }^{18} \mathrm{~F}$-fluorodeoxyglucose (FDG) positron emission tomography and computed tomography (PET/ $\mathrm{CT}$ ) is increasingly used in the diagnostic workup of infectious diseases. However, no large studies comparing the diagnostic value of ${ }^{18} \mathrm{~F}-\mathrm{FDG}$-PET/CT and MRI in patients with suspicion of spondylodiscitis have been performed. Small studies speculated that ${ }^{18} \mathrm{~F}$-FDG-PET/CT might have a higher sensitivity (up to 100\%) than MRI in diagnosing spondylodiscitis, especially in the early stages of disease. This might be explained by the different features of both imaging modalities, as MRI relies on anatomical changes, whereas ${ }^{18} \mathrm{~F}$-FDG-PET/CT visualizes glucose metabolism, which is already increased in the very early stages of inflammation [15].

Concerning the therapeutic strategy for infectious spondylodiscitis, there is no clear consensus, as so far no randomized clinical trials of short course or oral antibiotic regimens have been published [16]. Antibiotics are usually given intravenously for 4-6 weeks, including regimens against Staphylococcus aureus and possibly against Gram-negative bacteria until sufficient clinical improvement has been achieved, and CRP and ESR are reduced [17]. An additional oral course of at least 6 weeks is usually recommended [17].

Antimicrobial therapy is a very important issue in management of spondylodiscitis, whether or not surgical intervention is required [14]. In the current clinical case, initial antibiotic therapy with ceftriaxone and lincomycin which lasted 3 weeks was not sufficient, although the positive blood cultures led to targeting therapy. Pathogenic-specific antibiotic therapy should be given parenterally for 6-12 weeks [14]. Antibiotic therapy against methicillin-resistant Staphylococcus aureus was started for six consecutive weeks and rifampicin was prescribed for three months. After antimicrobial therapy clinical and radiological recovery was achieved, and no recurrence was detected in six-month follow-up of our case.

\section{Conclusions}

In conclusion, in any clinical aspect, infectious spondylodiscitis should be considered not only in patients with obvious risk factors. Also it is necessary to differentiate, in particular, with spondyloarthritis in the case of an unclear image of SpA, the severity of general symp- toms despite treatment, point spine pain and persistent inflammatory parameters in laboratory tests.

It is important to diagnose infectious spondylodiscitis early, and start the treatment as soon as possible considering the satisfactory response of spondylodiscitis to antimicrobial therapy in cases of early diagnosis.

The authors declare no conflict of interest.

\section{References}

1. Gouliouris T, Aliyu SH, Brown NM. Spondylodiscitis: update on diagnosis and management. J Antimicrob Chemother 2010; 65 (Suppl 3): iii11-24.

2. Fransen $\mathrm{BL}$, de Visser $\mathrm{E}$, Lenting $\mathrm{H}$, et al. Recommendations for diagnosis and treatment of spondylodiscitis. Neth J Med 2014; 72: 135-138.

3. Gottle L, Riordan T. Infectious spondylodiscitis. J Infect 2008; 56 : 401-412.

4. Diehn IE. Imaging of spine infection. Radiol Clin North Am 2012; 50: 777-798.

5. Skaf GS, Domlog NT, Fehlings MG, et al. Pyogenic spondylodiscitis; an overview. J Infect Public Health 2010; 3: 5-16.

6. Amiderfan K, McRoberts P, Deer TR. The differential diagnosis of low back pain: a primer on the evolving paradigm. Neuromodulation 2014; 17: 11-17.

7. Dufour V, Feydy A, Rillardon L, et al. Comparative study of postoperative and spontaneous pyogenic spondylodiscitis. Semin Arthritis Rheum 2005; 34: 766-771.

8. Legrand E, Flipo RM, Guggenbuhl PMC, et al. Management of nontuberculosis infectious discitis. Treatments used in $110 \mathrm{pa}-$ tients admitted to 12 teaching hospital in France. Joint Bone Spine 2001; 68; 504-509.

9. Demiraran Y, Dosoglu M, Yavuz C, Akyuz F. Spondylodiscitis and lumbar epidural abscess occurring after orthopedic epidural anesthesia: a case report. Turk Neurosurg 2006; 16: 208-211.

10. Vilke GM, Honingford EA. Cervical spine epidural abscess in a patient with no predisposing risk factors. Ann Emerg Med 1996; 27: 777-780.

11. Longo M, Granata F, Ricciardi K, et al. Contrast-enhanced MR imaging with fat suppression in adult-onset septic spondylodiscitis. Eur Radiol 2003; 13: 6262-627.

12. Lury K, Smith JK, Castillo M. Imaging of spinal infections. Semin Roentgenol 2006; 41: 363-379.

13. Ledermann HP, Schweitzer ME, Morrison WB, Carrino JA. MR imaging findings in spinal infections: rules or myths? Radiology 2003; 228: 506-514.

14. Gurbuz MS, Berkman MZ. Spondylodiscitis occurring after diagnostic lumbar puncture: a case report. Case Rep Infect Dis 2013; 2013: 843592.

15. Smids C, Kouijzer IJ, Vos FJ, et al. A comparison of the diagnostic value of MRI and ${ }^{18}$ F-FDG-PET/CT in suspected spondylodiscitis. Infection 2017; 45: 41-49.

16. Grados F, Lescure FX, Senneville E, et al. Suggestions for managing pyogenic (non-tuberculosis) discitis in adults. Joint Bone Spine 2007; 74: 133-139.

17. Kapsalaki E, Gatselis N, Stefos A, et al. Spontaneous spondylodiscitis: presentation, risk factors, diagnosis, management, and outcome. Intern J Infect Dis 2009; 13: 564-569. 